\title{
Early effects of parathyroid hormone on bisphosphonate/steroid-associated compromised osseous wound healing
}

\author{
S. Kuroshima • P. Entezami • L. K. McCauley • \\ J. Yamashita
}

Received: 24 May 2013 / Accepted: 19 August 2013 / Published online: 4 December 2013

(C) The Author(s) 2013. This article is published with open access at Springerlink.com

\begin{abstract}
Summary Administration of intermittent parathyroid hormone (PTH) promoted healing of tibial osseous defects and tooth extraction wounds and prevented the development of necrotic lesions in rats on a combined bisphosphonate and steroid regimen.

Introduction Osteonecrosis of the jaw (ONJ) has emerged in association with antiresorptive therapies. The pathophysiology of ONJ is unknown and no established cure currently exists. Our objective was to determine the effect of intermittent PTH administration on early osseous healing in the jaw and long bones of rats receiving bisphosphonate and steroid treatment.

Methods Ovariectomized rats received the combination therapy of alendronate and dexamethasone (ALN/DEX) for 12 weeks. Osseous wounds were created in the jaw and tibia. PTH was administered intermittently and healing at 2 weeks post-op was compared between the jaw and tibia by microcomputed tomography and histomorphometric analyses.

Results ALN/DEX treatment was associated with necrotic open wounds in the jaw but had no negative effects on healing and promoted bone fill in tibial defects. PTH therapy prevented the development of necrotic lesions in the jaw and
\end{abstract}

S. Kuroshima $\cdot$ J. Yamashita $(\bowtie)$

Department of Biologic and Materials Sciences, School of Dentistry,

University of Michigan, 1011 North University Ave., Ann Arbor,

MI 48109, USA

e-mail: yamashit@umich.edu

P. Entezami • L. K. McCauley

Department of Periodontics and Oral Medicine, School of Dentistry,

University of Michigan, Ann Arbor, MI, USA

L. K. McCauley

Department of Pathology, Medical School, University of Michigan,

Ann Arbor, MI, USA promoted healing of the tibial defects. PTH therapy was associated with the promotion of osteocyte survival in osseous wounds both in the jaw and tibia.

Conclusions Wound healing was impaired in the jaw in rats on a combined bisphosphonate and steroid regimen, and PTH therapy rescued necrotic lesions. These findings suggest that PTH therapy could be utilized to prevent ONJ from occurring in patients on combination antiresorptive and steroid therapy.

Keywords Alendronate $\cdot$ Bisphosphonate-associated osteonecrosis of the Jaw · Parathyroid hormone .

Wound healing

\section{Introduction}

Bone fracture and osteotomy cause osteocyte death which attracts osteoclasts for repair [1]. Osteoclasts are specialized cells responsible for bone resorption. During osseous wound healing, osteoclasts play an essential role in removing damaged bone and reshaping newly formed bone. Osteoclasts emerge in the early phase of osseous wound healing in long bones not only to resorb damaged bone but to also contribute to the orchestration of the entire repair process $[2,3]$. In the jaw soon after a tooth extraction, osteoclasts appear on the crestal bone area to resorb damaged bone $[4,5]$.

Nitrogen-containing bisphosphonates (N-BP), such as zoledronic acid and alendronate (ALN), are potent antiresorptives widely used for the management of bone metastatic diseases and osteoporosis. Recent reports have shown that antiresorptive therapy is associated with the development of osteonecrosis of the jaw (ONJ) [6]. ONJ is a rare and sitespecific complication related to potent antiresorptive therapy that uniquely occurs in the jaw [7]. The exact mechanism of this site specificity is not yet known. ONJ typically develops after invasive dental procedures such as tooth extractions in a 
small percent of patients with bone metastatic diseases receiving intravenous antiresorptive therapy [8]. These patients frequently have a history of steroid treatment and multiple chemotherapies. ONJ also occurs in patients taking oral antiresorptives for the management of osteoporosis; however, the incidence in this population is very low [9]. In the majority of patients taking oral antiresorptives, mucosal healing of tooth extraction sockets is uneventful even though osteoclastic bone resorption is hindered [10]. This may imply that osteoclast suppression alone is not sufficient to induce ONJ. Indeed, studies which investigated the effect of bisphosphonates on long bone fracture healing generally show increased callus formation, delayed callus remodeling, with no negative overall clinical impact on healing [11-13].

Parathyroid hormone (PTH) administered intermittently stimulates bone turnover and increases bone mass [14]. Teriparatide (rhPTH 1-34) is approved for the treatment of osteoporosis owing to its bone anabolic action [15]. Teriparatide has been reported to be associated with resolution of ONJ in several case reports [16] and shown to promote osseous healing in conjunction with oral surgery in humans [17]. Considering that N-BPs suppress, while PTH stimulates bone turnover, the resolution of ONJ and promotion of osseous healing by PTH therapy may be attributed to osteoclast activity.

Considering the number of patients taking bisphosphonates who may require a tooth extraction, a better understanding of the actions of bisphosphonates and PTH on extraction socket healing would lead to improved patient care. The goals of the present study were; (1) to determine the effect of the combination therapy of bisphosphonate and steroid prior to bone injuries on osseous healing, (2) to compare healing between tooth extraction sockets and tibial bony defects in bisphosphonate/steroid-treated rats, and (3) to investigate the effects of PTH therapy on early wound healing in bisphosphonate/steroid-affected bones. To achieve the study goals, ovariectomized-rats were treated with N-BP (ALN) and steroid (dexamethasone (DEX)), after which, bone injuries were created in the jaw and tibia. Early osseous wound healing with and without daily PTH was assessed using microcomputed tomography (microCT) and histology and results compared.

\section{Material and methods}

Animals and in vivo injections

The experimental protocol was approved by the University Committee on Use and Care of Animals. Female Sprague Dawley rats ( 9 weeks, $n=28$ ) were maintained at $22{ }^{\circ} \mathrm{C}$ in 12-h light/12-h dark cycles and allowed free access to water and standard rodent diet. All rats underwent bilateral ovariectomy (OVX) at 10 weeks of age to induce estrogendeficient bone loss experimentally. A bisphosphonate (ALN) and DEX were subcutaneously administered to induce necrotic lesions in tooth extraction wounds [18, 19]. The ALN (Sigma-Aldrich, St. Louis, MO) treatment was initiated at the time of OVX. ALN was administered $(0.8 \mathrm{mg} / \mathrm{kg})$, twice a week for 12 weeks to half of the rats as well as daily DEX treatment (Tocris, Ellisville, MO) at $1 \mathrm{mg} / \mathrm{kg}$ for the last 2 weeks. The other half of rats received vehicle (saline) as control. The subcutaneous DEX and ALN dosages were calculated based on the body surface area normalization method [20] and correspond to the human systemic DEX dose (10 mg/ day) and approximately $20 \%$ of the human oral ALN dose (70 $\mathrm{mg} /$ week). At the end of the ALN and DEX (or vehicle) administration, maxillary right second molars (M2) were extracted and osseous defects created in the tibia and jaw. Post tooth extractions, half of ALN/DEX-treated rats and VC-rats further received daily PTH injections (Bachem, Torrance, CA) at $80 \mu \mathrm{g} / \mathrm{kg}$ for 2 weeks and the other half daily saline injections. Hence, a total of four groups ( $n=7 /$ group) was established (A/D-VC, A/D-PTH, VC-VC, and VC-PTH; Fig. 1a). All rats were euthanized 2 weeks post-extractions of tooth.

Tibial bone defects and tooth extractions

The osseous defects were created in the tibia and jaw (Fig. 1b, c) under ketamine/xylazine general anesthesia. An $\sim 10$-mm incision was made below the knee joint. The periosteum was reflected to expose the bone surface distal to the proximal metaphysis. A hole ( $1 \mathrm{~mm}$ in diameter) was drilled at $4.5 \mathrm{~mm}$ distal to the growth plate using a round bur $<2,000 \mathrm{rpm}$ under copious irrigation. Primary closures were achieved using surgical staples. Next, the maxillary right second molar was extracted using dental instruments. The extraction sites were left as open wounds as clinically performed in human tooth extractions.

\section{Microcomputed tomography}

At killing, the maxillae and tibiae were dissected, fixed in $10 \%$ formalin, and analyzed using microCT ( $\mu \mathrm{CT}-100$; Scanco Medical AG, Bruttisellen, Switzerland) to assess bone parameters in extraction sockets and tibial defects as well as in intact bone. The maxillae and tibiae were scanned at $10-\mu \mathrm{m}$ voxel resolution with an energy level of $70 \mathrm{kV}$. The extraction sockets were segmented by the semi-manual contouring method [21]. The tibial defects were scanned from 3.7 to $5.9 \mathrm{~mm}$ distal to the growth plate (Fig. 1b). To establish baseline bone responses to the treatment, the metaphyseal bone of the proximal tibiae from 1.2 to $3.5 \mathrm{~mm}$ distal to the growth plate and the interradicular bone between the mesial and distal roots of 


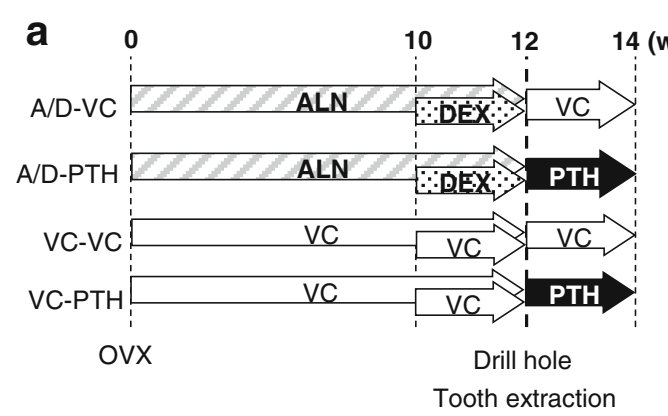

Fig. 1 Experimental schedule. a Rats $(n=14)$ received ALN for 12 weeks and dexamethasone for 2 weeks before tooth extraction and osseous defect surgeries. Another14 rats received vehicle control (saline). Immediately after the surgeries, half of rats in each group received daily PTH administration $(80 \mu \mathrm{g} / \mathrm{kg})$ for 2 weeks and the remaining half vehicle control. b MicroCT scanning was performed in the proximal tibiae between 1.2 and $3.5 \mathrm{~mm}$ from the growth plate to determine the

the maxillary first molars (Fig. 1c) were assessed as well [22]. Data were analyzed using the built-in Scanco software.

\section{Histomorphometry}

The maxillae and tibiae were demineralized in $10 \%$ ethylenediaminetetraacetic acid at $4{ }^{\circ} \mathrm{C}$, paraffin embedded, and sectioned at $5 \mu \mathrm{m}$. Hematoxylin and eosin, tartrate resistant acid phosphatase, and Masson's trichrome staining was performed following standard protocols and/or manufacturer's instructions (386A, HT15, Sigma-Aldrich, St. Louis, MO) [23]. Blood vessels in the extraction wounds were immunohistochemically stained. Briefly, sections were deparaffinized, nonspecific protein blocked, and incubated overnight with a rabbit von Willebrand factor (vWF) antibody (ab6999, Abcam, Cambridge, MA). Goat anti-rabbit IgG (AP307, Millipore) was used as a secondary antibody and proteins developed with 3,3-diaminobenzidine (Vector Laboratories, Burlingame, CA). Sections were counterstained with hematoxylin. Terminal deoxynucleotidyl transferase dUTP nick end labeling (TUNEL) staining was performed to assess apoptotic cells in the tibial defects (FragEL ${ }^{\text {TM }}$ DNA Fragmentation Detection Kit, EMD Chemicals, Gibbstown, NJ). Empty osteocyte lacunae were quantified within $100 \mu \mathrm{m}$ depth of bone surface. Necrotic bone area, defined as the portion of bone with greater than or equal to 10 adjacent empty osteocyte lacunae, was measured [24]. Polymorphonuclear cell (PMN) infiltration was assessed by quantifying inflammatory cell numbers in soft tissue within $100 \mu \mathrm{m}$ of the bone surface (area of interest (AOI), $0.1 \times 2.5 \mathrm{~mm}$ ). Collagen deposition and $\mathrm{vWF}^{+}$blood vessels were assessed in the soft tissue next to the bone surface (AOI, $0.4 \times 2.5 \mathrm{~mm}$ ). All histomorphometric analyses were performed using Image-Pro (Media Cyberrnetics, Bethesda, MD).
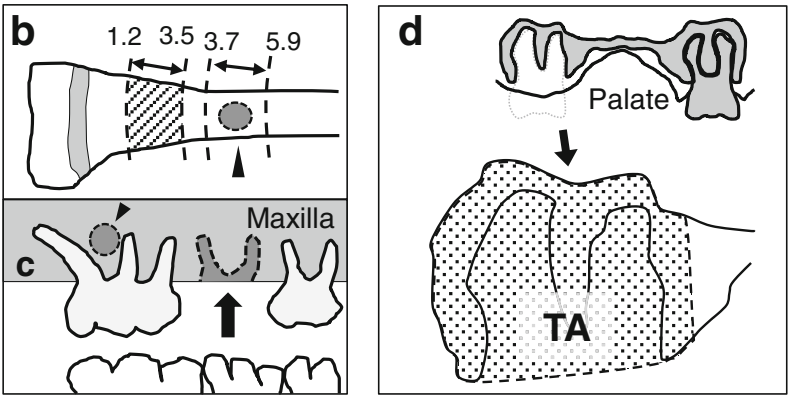

treatment effect on undisturbed trabecular bone. Scanning between 3.7 and $5.9 \mathrm{~mm}$ away from the growth plate was used to asses osseous healing (arrowhead). c The microCT scanning sites in the maxillae: tooth extraction wounds (arrow) and the interradicular bone (arrowhead) of the neighboring tooth. d A schematic drawing of a tooth extraction socket. Tissue area (TA) was defined as the area of an extraction socket and surrounding bone

Statistics

Statistical analysis was conducted with SYSTAT 12 (Systat Software, Chicago, IL) and InStat (GraphPad Software, San Diego, CA). Analysis of variance was performed for multiple groups with a Tukey's post hoc test. For comparisons within the group, paired $t$ test was conducted. The PTH effect on the mucosal wound closure was assessed using Fisher's exact test. An $\alpha$-level of 0.05 was used for statistical significance. Results are presented as mean \pm SEM unless specified.

\section{Results}

PTH actions in intact tibiae were greatest in rats treated with ALN/DEX

Bone volume and bone mineral density (BMD) in the intact tibial metaphysis were significantly higher in the ALN/DEX treatment groups vs. vehicle control (Fig. 2a-f). PTH following ALN/DEX showed a non-significant trend toward higher bone volume and BMD versus ALN/DEX-VC. PTH had little bone anabolic effect in the group without the ALN/DEX treatment. However, trabecular thickness was significantly higher in the VC-PTH vs. control (Fig. 2d). Interestingly, the bone anabolic effect of PTH was more pronounced after ALN/ DEX than after VC treatment in the intact tibial metaphysis (Fig. 2g).

PTH actions in wounded tibiae were blunted in rats treated with ALN/DEX

In the tibial wounds, bone fill and BMD were significantly higher in the ALN/DEX treatment groups vs. vehicle control (Fig. 3a-f). PTH significantly enhanced bone fill, trabecular 
Fig. 2 Treatment effect on undisturbed bone. a

Representative longitudinal and cross-sectional images of the undisturbed tibiae. The ALN/ DEX treatment resulted in significantly higher bone mass (b), trabecular numbers (c), BMD (f), and lower trabecular separation (e) compared with the $\mathrm{VC}$ treatment groups. PTH for 2 weeks significantly increased trabecular thickness regardless of the treatment (d). A nonsignificant increase by PTH was noted in bone mass (b) and $\mathrm{BMD}$ (f) in the ALN/DEX treatment group. When the bone mass increase by PTH was compared between the ALN/ DEX and VC treatment groups, a significantly greater increase was noted in the ALN/DEX treatment group (g). * $p<0.05 ; * * p<0.01$; $* * * p<0.001$ versus control $(V C-V C)$

a

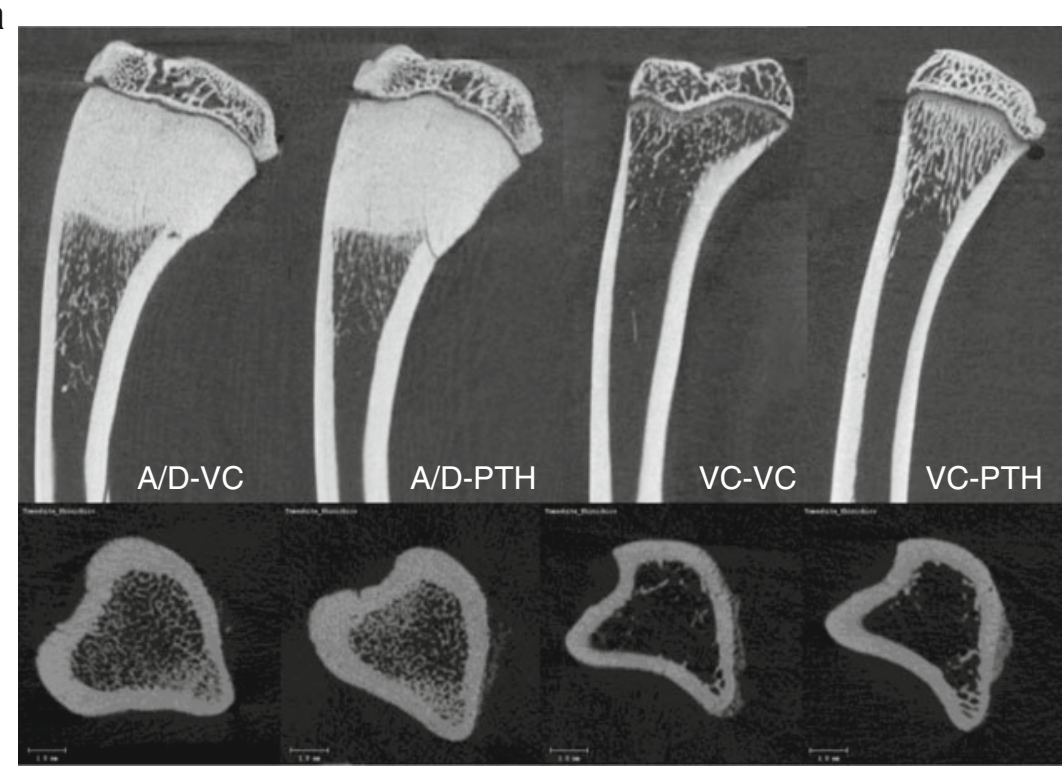

b

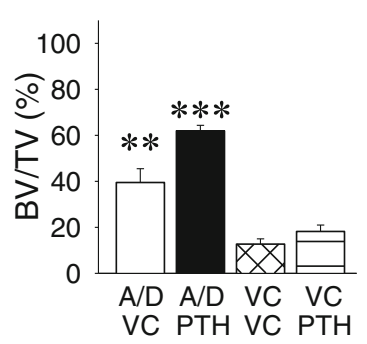

e

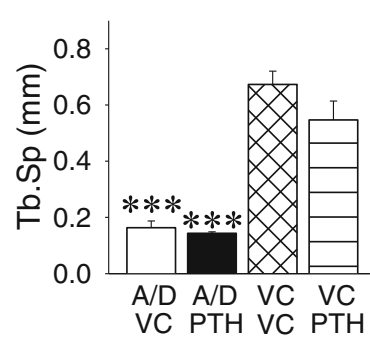

C

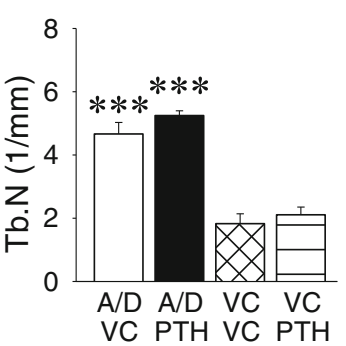

f

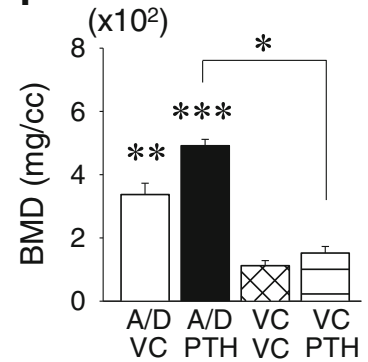

d $\left(x 10^{-1}\right)$

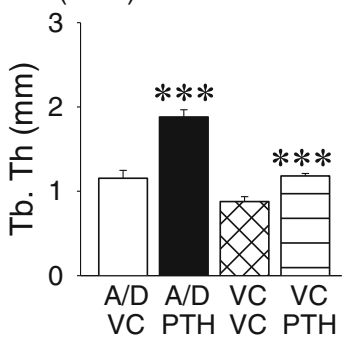

g

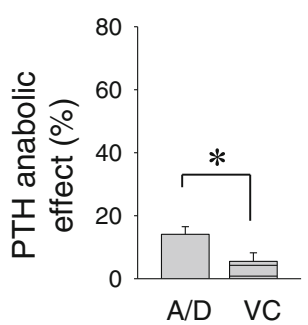

thickness, and BMD regardless of the presence or absence of the ALN/DEX treatment. The PTH effect observed in wounded controls was very different from that observed in the intact tibiae (Figs. 3b vs. 2b). The bone anabolic effect of PTH was significantly more robust after the VC than after ALN/DEX treatment (Fig. 3g), suggesting that the ALN/DEX treatment had a restrictive impact on the PTH anabolic effect in the tibial osseous wounds.

PTH promoted osteocyte and bone marrow cell survival in tibial wounds

Healing of the tibial wounds was further assessed in histologic sections. Tissue area (TA) was defined as the area surrounded by the cortical bone (Fig. 4a). Bone fill (bone area (BA)/TA) was significantly higher in the ALN/DEX treatment groups versus vehicle control (Fig. 4b). Significantly higher bone fill was noted in the PTH-treated groups irrespective of the presence or absence of the ALN/DEX treatment. These results were consistent with those of the microCT assessment (Fig. 3b). Periosteal callus formation was observed in the ALN/DEX-PTH group but statistical significance was not reached (Fig. 4c). The ALN/DEX treatment significantly reduced osteoclast surface compared with control with a substantial reduction by PTH following ALN/DEX (Fig. 4d). Osteoblast surface was not affected by the ALN/DEX treatment but PTH resulted in significantly higher osteoblast surface than VC following ALN/DEX (Fig. 4e). The incidence of 
Fig. 3 Treatment effect on the tibial defects. a Representative cross-sectional images of the tibial defects. Regardless of treatment, significantly higher bone mass (b), trabecular numbers (c), BMD (f), and lower trabecular separation (e) were noted in the treatment groups vs. control. PTH significantly increased trabecular thickness in the ALN/DEX and VC treatment groups but the ALN/DEX treatment alone had no effect on trabecular thickness (d).

Although PTH further increased bone mass (b) and BMD (f) after the ALN/DEX treatment, an average bone mass increase by PTH was significantly less after ALN/DEX compared with VC (g). ${ }^{* * *} p<0.001$ versus control $(V C-V C) ;{ }^{\dagger \dagger} p<0.001$ versus the ALN/DEX-VC group

a

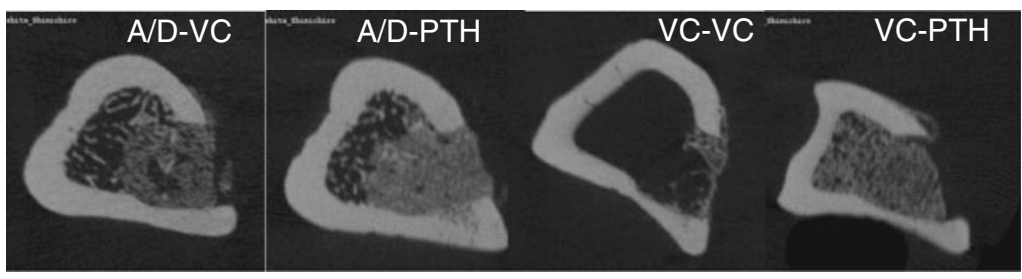

b

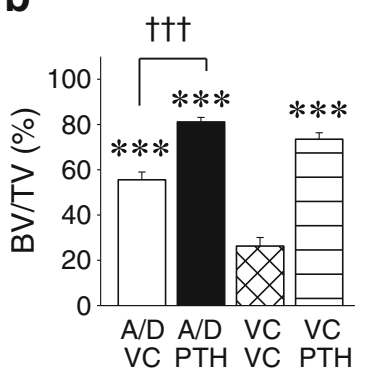

e

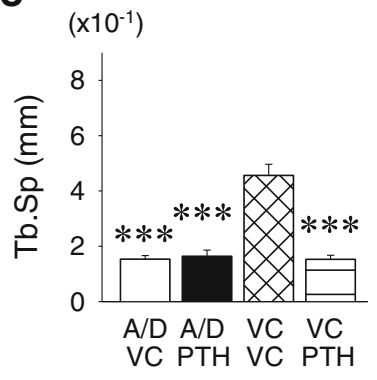

c

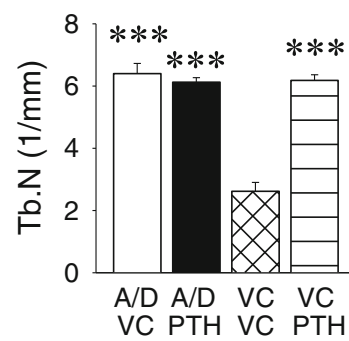

$f$

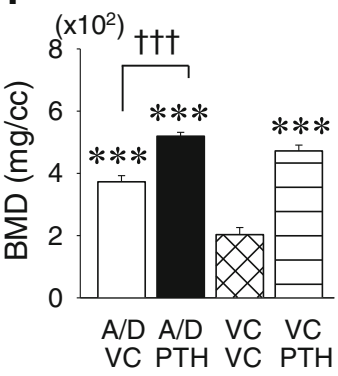

d

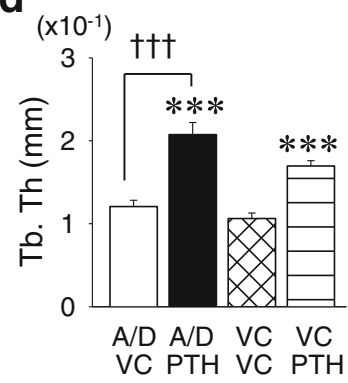

g

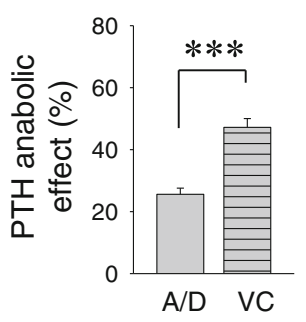

empty osteocyte lacunae and necrotic bone were significantly lower in PTH-treated groups regardless of the presence or absence of the ALN/DEX treatment (Fig. 4f, g), suggesting that PTH promoted osteocyte survival. Apoptotic bone marrow cells in the defects were visualized with TUNEL staining and histomorphometrically assessed. PTH significantly reduced numbers of TUNEL-positive apoptotic bone marrow cells compared with control irrespective of the presence or absence of the ALN/DEX treatment (Fig. 4h).

PTH promoted tooth extraction wound healing after ALN/DEX treatment

Next, treatment effects were assessed in the jaw. In the interradicular bone near the tooth extraction site (Fig. 1c), the ALN/DEX treatment resulted in significantly higher bone volume and BMD than control (Fig. 5a). The average bone anabolic effect of PTH was significantly higher in the VC than ALN/DEX treatment group. In the tooth extraction sockets, bone fill and BMD were significantly higher in the ALN/DEX treatment groups than controls (Fig. 5b). PTH significantly enhanced bone fill and BMD regardless of the presence or absence of the ALN/DEX treatment. However, the average PTH bone anabolic effect was significantly less in the ALN/
DEX vs. VC treatment group, again indicating that the ALN/ DEX treatment suppressed bone anabolism by PTH in the tooth extraction wounds.

PTH rescued ALN/DEX-associated deterred tooth extraction wound healing

Tooth extraction wound healing was assessed histomorphometrically. Representative photomicrographs of the trichrome-stained sections of the tooth extraction wounds at 2 weeks post-extractions are shown in Fig. 6a. Open wounds with exposed bone were noted in six rats in the ALN/DEX-VC group and 1 rat in the ALN/DEX-PTH group. In vehicle control (VC-VC), three rats showed open wounds, while no open wound was noted in the VC-PTH group. PTH administration after tooth extractions promoted healing regardless of the presence or absence of the ALN/DEX treatment with significant improvement after the ALN/DEX treatment. TA was defined as the portion of the maxilla surrounding the roots of M2 (Fig. 1d) and bone area (BA/TA) was assessed. The histomorphometric assessment revealed significantly higher bone area in the ALN/DEX-VC, ALN/DEXPTH and VC-PTH groups vs. control (Fig. 6b), which was consistent with the microCT findings (Fig. 5b). The ALN/ 
Fig. 4 Histomorphometric assessments of tibial wound healing. a A diagram of the crosssectional view of a tibial defect indicating the tissue area (TA). Both the ALN/DEX and PTH treatment resulted in significantly higher bone area vs. control (b). PTH after the ALN/DEX treatment significantly increased bone area. No differences were noted in periosteal callus formation between groups, but a trend of more periosteal callus in the ALN/DEX-PTH group vs. control was observed (c). The ALN/DEX treatment significantly suppressed osteoclast surface vs. control with further significant reduction in the ALN/DEX-PTH group (d). The ALN/DEX treatment had no effect on osteoblast surface vs. control. PTH significantly increased osteoblast surface after ALN/DEX (e). PTH therapy significantly suppressed the numbers of empty osteocyte lacunae and necrotic bone area regardless of other treatment (f, g). $\mathrm{TUNEL}^{+}$cells in the bone marrow were significantly reduced by PTH compared with control (h). ${ }^{*} p<0.05 ; * * p<0.01$; $* * * p<0.001$ versus control $(V C$ $V C) ;{ }^{\dagger} p<0.05 ;{ }^{\dagger \dagger} p<0.01$ versus the ALN/DEX-VC group a

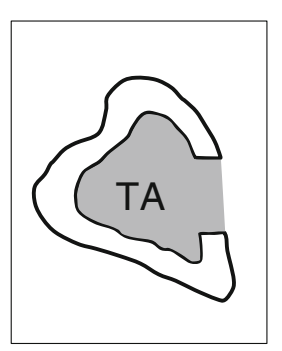

b

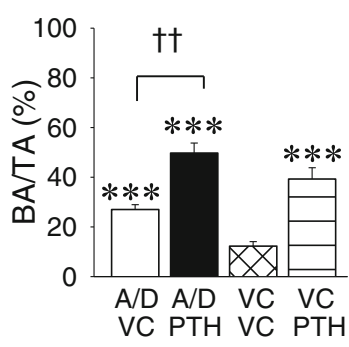

d

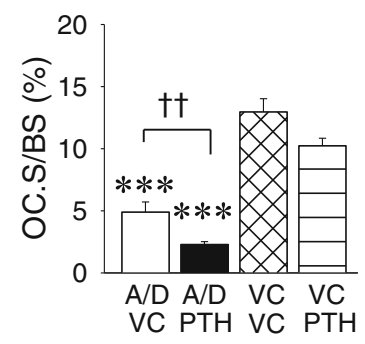

g

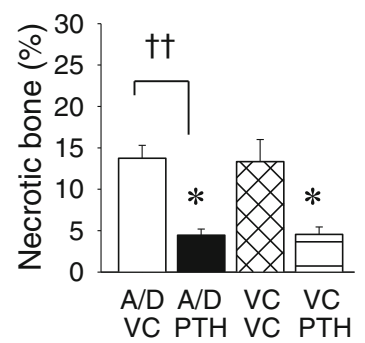

h
C

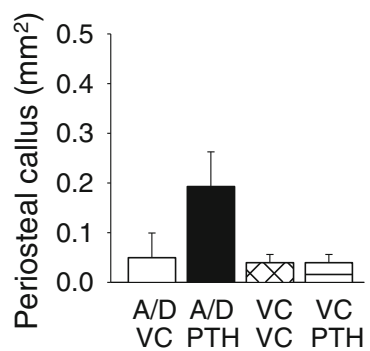

f
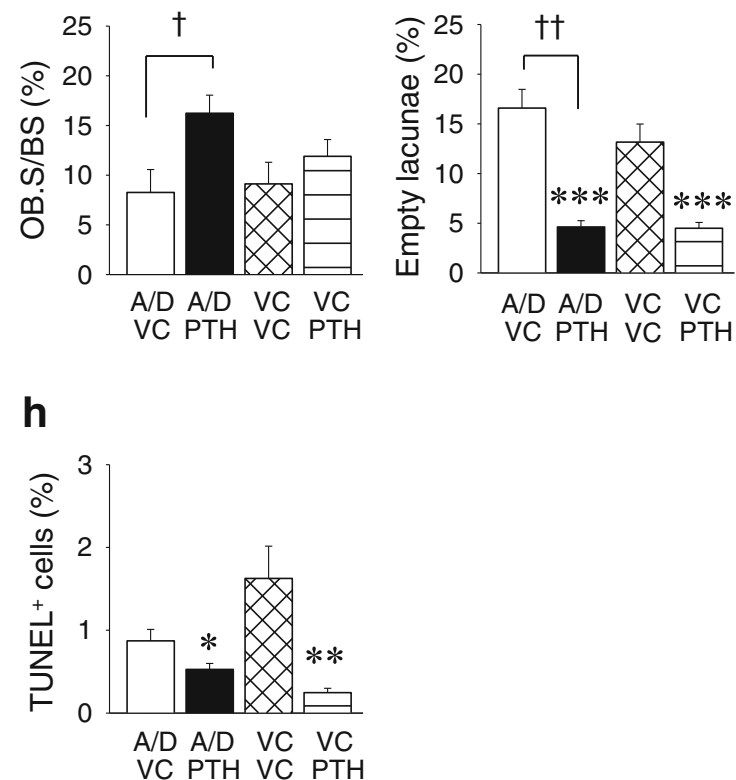

DEX treatment significantly suppressed osteoclast surface compared with control (Fig. 6c). PTH resulted in significantly higher osteoclast surface after the VC treatment but not after the ALN/DEX treatment. Likewise, significantly higher osteoblast surface by PTH was noted after the VC treatment but not after the ALN/DEX treatment (Fig. 6d). The ALN/DEX treatment had no apparent effect on osteoblast surface. The numbers of empty osteocyte lacunae and necrotic bone were significantly higher in the ALN/DEX-VC group versus control (Fig. 6e, f). PTH significantly reduced the numbers of empty osteocyte lacunae and presence of necrotic bone. Similarly, PTH significantly reduced the numbers of empty lacunae and necrotic bone when the VC-VC and VC-PTH groups were compared, suggesting that PTH promoted osteocyte survival. PMN infiltration was also significantly higher in the ALN/DEX-VC group and PTH significantly reduced PMNs in the ALN/DEX-PTH group (Fig. 6g). Connective tissue maturation as measured by collagen apposition was lower in the ALN/DEX-VC group vs. control (Fig. 6h); however, PTH significantly enhanced the collagen apposition regardless of presence or absence of the ALN/DEX treatment.
There were no differences noted in the numbers of blood vessels between groups (Fig. 6i).

\section{Discussion}

The ALN/DEX treatment resulted in high bone mass in both the tibia and jaw as anticipated [26]. However, its effect on osseous wound healing was distinct; the ALN/DEX treatment enhanced early osseous healing in the tibial wounds by increasing bone fill, while it impaired tooth extraction wound healing with exposed bone. The effect of bisphosphonate therapy on osseous healing in rat long bones has previously been reported in the literature $[26,27]$. Those reports agree that bisphosphonate therapy promotes osseous repair by enhancing formation, mineralization, and mechanical strength of callus, but also slows callus remodeling. Hence, our result of high bone fill by ALN/DEX is consistent with the literature. Despite the positive impact on tibial wound healing, in contrast, ALN/DEX impaired tooth extraction wound healing in the jaw and resulted in a greater incidence of exposed bone. 


\section{a Interradicular bone}
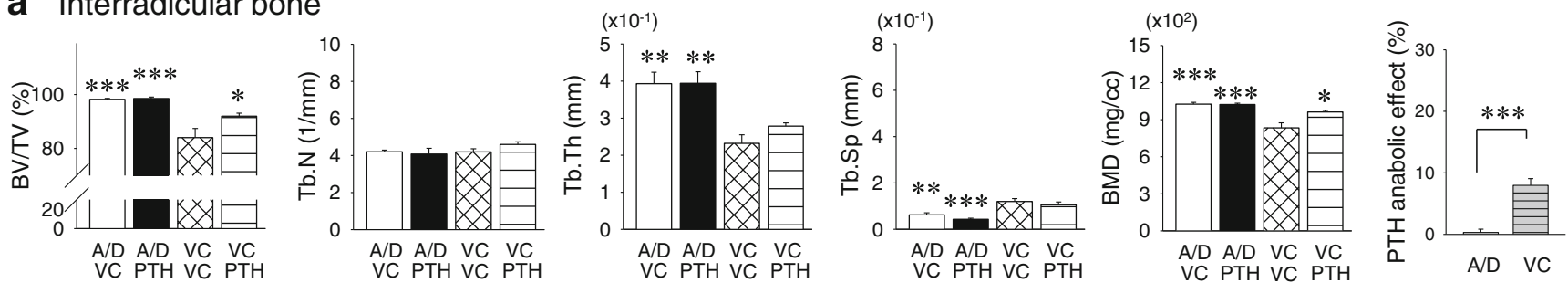

b Tooth extraction sockets
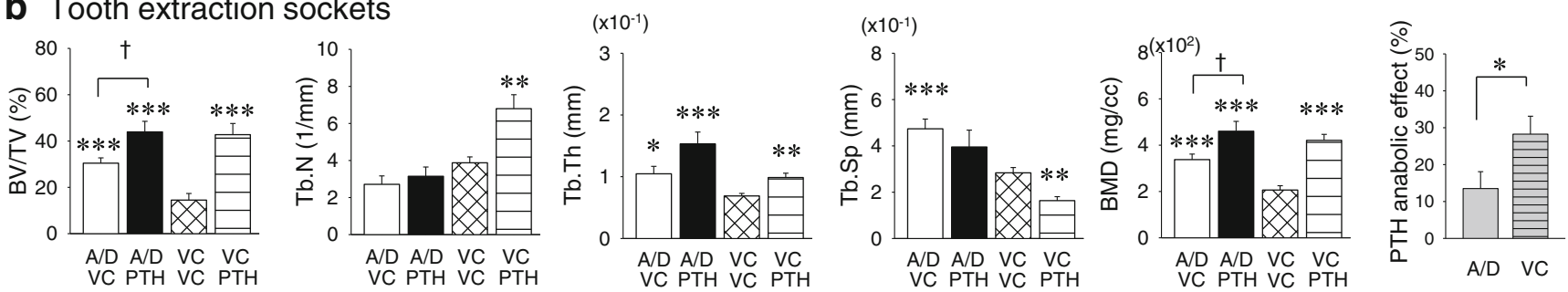

Fig. 5 Treatment effect on the maxillae. a The result of microCT assessment of undisturbed maxillae. Regardless of treatment, significantly higher bone mass and BMD were found in the treatment groups vs. control. The ALN/DEX treatment significantly increased trabecular thickness and decreased trabecular separation compared with control. No PTH anabolic effect was observed after the ALN/DEX treatment, while PTH increased bone mass significantly after VC. $\mathbf{b}$ The result of

The combined use of bisphosphonates and steroid has been demonstrated to be associated with the development of necrotic lesions in rats $[18,19]$. The impaired extraction wound healing by ALN/DEX observed in our study is consistent with these reports. It should be mentioned that although the bisphosphonate/steroid treatment impaired tooth extraction wound healing in rats, such a drug combination does not always hinder wound healing in other animals [28].

The difference in osseous healing between the tibia and jaw may be similar to what is seen in patients on antiresorptive therapy. ONJ uniquely occurs in the jaw but not in long bones [29]. Tooth extraction wounds are different from tibial osseous wounds in that (1) they are open wounds exposed to the oral cavity where numerous oral pathogens inhabit and dense bacterial colonization occurs [30], (2) the extraction wounds are subjected to repeated mechanical trauma from chewing, (3) the extraction sockets are surrounded by dense bundle bone while the tibial wounds are exposed to the abundance of the bone marrow milieu, (4) the embryologic origin of the maxillae and mandibles (pharyngeal arch 1) is distinct from long bones [31], and (5) the bone formation pattern of the alveolar bone is different from that of long bones (intramembranous vs. endochondral bone formation) [32]. Considering these differences, tooth extraction wound healing appears to be distinct from long bone wound healing. However, the exact mechanism of the different healing responses between the tibia and jaw is unclear. The etiopathological role of oral bacteria in ONJ has been proposed; when bacterial infection, such as periodontitis, was experimentally induced in rats receiving bisphosphonates, necrotic lesions developed,
microCT assessment of tooth extraction wounds. Both the ALN/DEX and PTH treatments resulted in significantly higher bone mass, trabecular thickness, and BMD than control. Although PTH significantly increased bone mass and BMD after ALN/DEX, an average increase in bone mass by PTH was significantly smaller after ALN/DEX than VC. ${ }^{*} p<0.05$; ${ }^{* *} p<0.01 ; * * * p<0.001$ versus control $(V C-V C) ;{ }^{\dagger} p<0.05$ versus the ALN/DEX-VC group

however, no such lesions occurred in rats without bisphosphonate therapy [33, 34]. In support of this hypothesis, LopezJornet et al. reported that antibiotic administration prior to tooth extractions in rats on the combination of bisphosphonates and DEX significantly reduced the incidence of necrotic lesions [35]. Whether bisphosphonate treatment exacerbates bacterial infection or not was studied using a rat model of infectious osteomyelitis [36]. In this study gentamycin-sensitive Staphylococcus aureus -treated implants were placed in rat tibiae with or without ALN treatment. Highgrade infection and necrotic bone formation were found with ALN treatment, while neither infection nor necrotic bone was noted with placebo. Gentamycin therapy ameliorated the infection and resulted in no necrotic bone formation. Although the study was osteomyelitis focused, the findings support the etiopathological role of bacteria in ONJ.

In the current study, intermittent PTH administration for 2 weeks after $\mathrm{VC}$ treatment resulted in significantly higher bone mass in intact maxillae but not in intact tibiae. The difference in bone responses to PTH is likely due to the presence or absence of trabecular bone. In this study, the metaphyseal trabecular bone area between 1.2 and $3.5 \mathrm{~mm}$ distal to the growth plate was assessed to establish baseline bone responses to PTH. As the assessed bone site corresponds to the distal end of the metaphyseal trabecular bone in the proximal tibiae, the trabecular bone at this site would be resorbed because of OVX in the VC-treated rats. Accordingly, the trabeculation was scarce when PTH therapy was initiated. The relatively high BMD values of the maxillae in the $\mathrm{VC}-\mathrm{VC}$ group suggests the trabecular structure was maintained after OVX, while in the tibiae the low BMD values in the VC-VC 
a
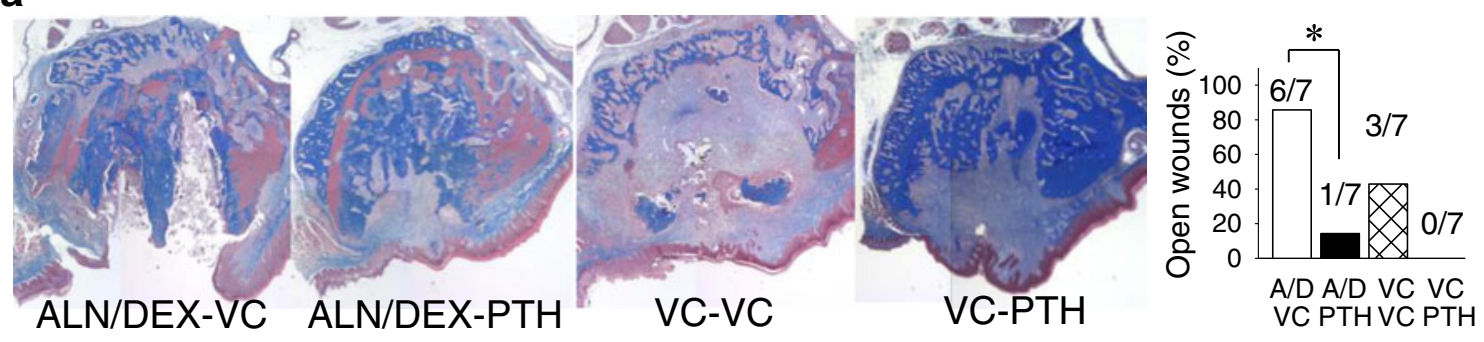

b

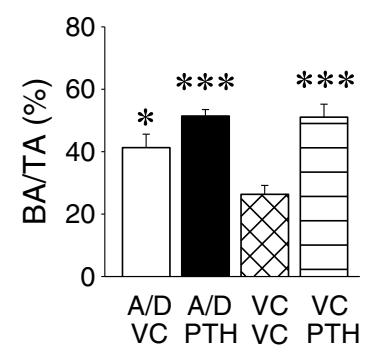

f

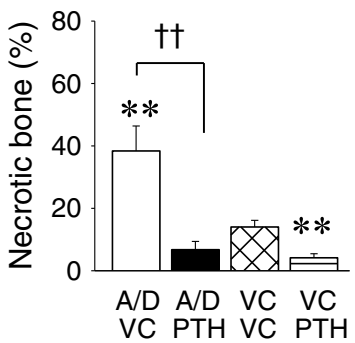

C

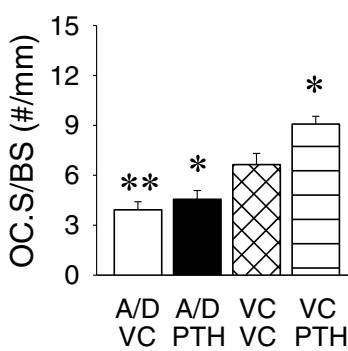

$9\left(\times 10^{2}\right)$

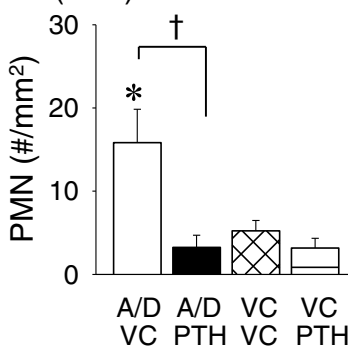

d

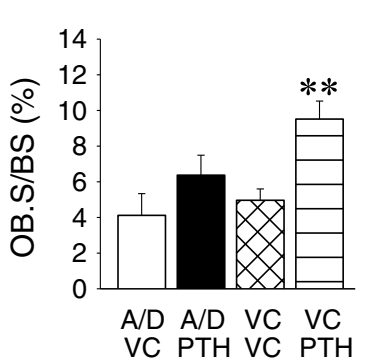

h

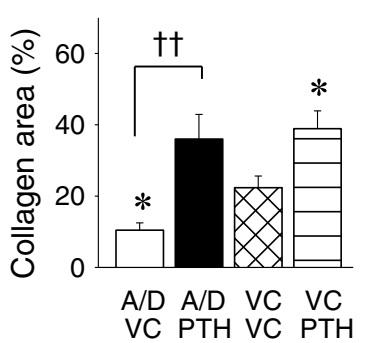

e

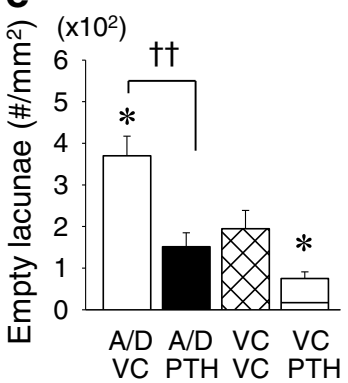

i

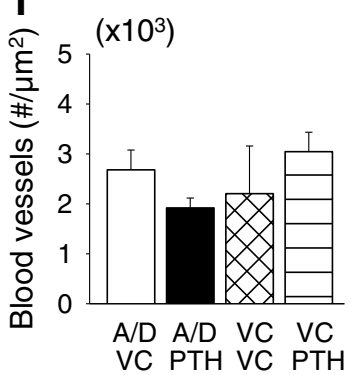

Fig. 6 Histomorphometric assessments of extraction wound healing. (a) Representative images of frontal-sections of the extraction wounds. Six rats developed necrotic lesions in the ALN/DEX-VC group and only one in the ALN/DEX-PTH group. Both the ALN/DEX and PTH treatments resulted in significantly higher bone area vs. control (b). The ALN/DEX treatment significantly suppressed, and PTH after VC, significantly increased osteoclast surface (c). PTH significantly increased osteoblast surface after VC but not after ALN/DEX (d). Significantly higher numbers of empty osteocyte lacunae and necrotic bone area were noted in the ALN/DEX-VC group vs. control. PTH suppressed the numbers of empty

lacunae and necrotic bone area significantly after ALN/DEX and after VC $(\mathbf{e}, \mathbf{f})$. PMN infiltration was significantly higher in the ALN/DEX-VC group versus control. PTH dramatically suppressed PMN infiltration when given after ALN/DEX (g). Significantly lower collagen apposition was noted in the ALN/DEX-VC group vs. control. PTH increased collagen apposition significantly after ALN/DEX and after VC (h). No treatment regimen altered blood vessel numbers (i). ${ }^{*} \mathrm{p}<0.05 ;{ }^{*} \mathrm{p}<$ $0.01 ; * * * \mathrm{p}<0.001$ versus control $(\mathrm{VC}-\mathrm{VC}) ; \mathrm{\dagger p}<0.05 ; \dagger \dagger \mathrm{p}<0.01$ versus the ALN/DEX-VC group

group points to significant trabecular bone loss. Therefore, in the intact tibiae that the PTH anabolic effect was not observed was likely due to a trabeculation deficit. Rats in which ALN/DEX treatment was initiated immediately after OVX had greater trabecular bone as evidenced by the high BV/TV and BMD values in the ALN/DEX-VC group. In the ALN/DEX-treated rats, PTH therapy augmented BV/TV and BMD. In fact, when the PTH anabolic effect was compared between ALN/DEX and VC treatment, significantly higher bone volume was found in the ALN/DEXtreated rats. These findings may suggest that the amount of existing trabecular bone is a determinant of the degree of PTH anabolic effect in the metaphysis. It is also possible that the short duration (2 weeks) of PTH treatment was not long enough to support significant anabolism at this site. The tibial bone defects were made at the edge of the diaphysis where little trabecular bone, if any, existed. Even the defects were created in such a sparse trabecular bone area in the VC-treated rats, PTH significantly promoted bone fill. PTH also enhanced bone fill in the defects significantly after the ALN/DEX treatment. When the PTH anabolic effect was compared between the osseous defects and undisturbed bone, more powerful PTH anabolic effect was noted in the osseous defect than in undisturbed bone in this study (approximately 47 vs. $6 \%$ ). PTH has been shown to promote osseous healing in osteoporotic women [37]. The PTH anabolic effect has also been shown to be pronounced in rapidly growing animals [38]. Nakajima et al. reported that low doses of PTH, which did not increase systemic bone mass, was sufficient to promote osseous healing in rats [39]. These reports together with our findings 
suggest that PTH's anabolic actions are greatly enhanced in bone with a high metabolic state. It should be mentioned that although PTH significantly augmented bone fill in the defects after ALN/ DEX, this increase was smaller than that after VC treatment, suggesting a possible blunting effect of ALN/DEX treatment on the PTH anabolic effect [40].

In extraction wounds, PTH rescued ALN/DEX-induced impaired healing evidenced by high bone fill and promotion of soft tissue coverage. PTH's ability to promote healing of ONJ in osteoporotic patients has been reported in case studies [16], however, its mechanisms are unknown. Our study may provide a biological explanation. In the current study, the ALN/DEX treatment significantly suppressed osteoclasts in the extraction wounds. Osteoclast recovery, however, appeared to not be critical for healing since osteoclast surface was significantly suppressed in the healed wounds of the ALN/DEX-PTH group. Rather, the reduction of empty osteocyte lacunae appeared to be associated with healing. PTH significantly reduced the empty lacunae in both the ALN/ DEX- and VC-treated rats, suggesting that PTH may promote osteocyte survival in extraction wounds. The significant reduction in empty osteocyte lacunae was observed not only in the extraction wounds but also in the tibial defects. In the tibial defects, it is likely that the surgical drill created damage in the bone and induced osteocyte death. PTH significantly promoted osteocyte survival in both the ALN/DEX and VC-treated groups. Furthermore, PTH appeared to promote the survival of bone marrow cells as suggested by the numbers of $\mathrm{TUNEL}^{+}$bone marrow cells that were significantly suppressed by PTH in the tibial defects. Intermittent PTH is known to have antiapoptotic effects in mature osteoblasts [41], but our findings suggest that PTH might have antiapoptotic effects on other cell types including osteocytes in osseous wounds. In this study, PTH suppressed PMN infiltration and promoted collagen apposition significantly in the extraction wounds. Although unclear, we speculate that the suppression of osteocyte death by PTH reduced inflammatory responses and therefore suppressed PMN infiltration, and such a diminution in inflammatory responses promoted soft tissue healing by increasing collagen apposition.

Abtahi et al. compared the incidence of necrotic lesions with and without wound coverage post-extractions in rats on ALN/ DEX and found that all extraction wounds developed necrotic lesions when the wounds were left open, but with the wound coverage, no necrotic lesions occurred [42]. In the present study, the tooth extraction wounds were left open, while the tibial defects were closed. Extraction wounds are typically left open in humans, so it is possible that if the oral wounds were closed in this study, they could have healed in a similar manner to the tibial wounds. The observed differences in this study could be, therefore, to a small extent attributed to the presence or absence of wound closure. Rats heal rapidly after tooth extraction; epithelial coverage occurs in approximately 8 days and bone fill happens in approximately 3 weeks [5]. Assessment of healing at 2 weeks was chosen since a primary goal for this study was to evaluate short-term actions of PTH during the course of wound healing. However, this time period could fall short and the outcome of this study may be different if PTH therapy had been extended.

This study shows that ALN and DEX treatment restricted tooth extraction wound healing in the jaw. Intermittent PTH rescued bisphosphonate/dexamethasone-induced necrotic lesions by promoting soft tissue healing. The findings of this study suggest that intermittent PTH therapy could be considered to prevent $\mathrm{ONJ}$ in osteoporosis patients receiving ALN and steroid therapies.

Acknowledgments This work was supported by a 2012 Award from the Delta Dental Foundation, the NIH/NIDCR R01DE023538, and R01DE022327. The MicroCT core is funded in part by NIH/NCRR S10RR026475.

Conflicts of interest Dr. McCauley is a co-investigator on a human clinical trial where Eli Lilly provided study drug.

Open Access This article is distributed under the terms of the Creative Commons Attribution Noncommercial License which permits any noncommercial use, distribution, and reproduction in any medium, provided the original author(s) and the source are credited.

\section{References}

1. Verborgt O, Gibson GJ, Schaffler MB (2000) Loss of osteocyte integrity in association with microdamage and bone remodeling after fatigue in vivo. J Bone Miner Res 15:60-67

2. Schell H, Lienau J, Epari DR, Seebeck P, Exner C, Muchow S, Bragulla H, Haas NP, Duda GN (2006) Osteoclastic activity begins early and increases over the course of bone healing. Bone 38:547-554

3. Clark WD, Smith EL, Linn KA, Paul-Murphy JR, Muir P, Cook ME (2005) Osteocyte apoptosis and osteoclast presence in chicken radii 0-4 days following osteotomy. Calcif Tissue Int 77:327-336

4. Pietrokovski J, Massler M (1971) Residual ridge remodeling after tooth extraction in monkeys. J Prosthet Dent 26:119-129

5. Smith N (1974) A comparative histological and radiographic study of extraction socket healing in the rat. Aust Dent J 19:250-254

6. Ruggiero SL, Mehrotra B, Rosenberg TJ, Engroff SL (2004) Osteonecrosis of the jaws associated with the use of bisphosphonates: a review of 63 cases. J Oral Maxillofac Surg 62:527-534

7. Saad F, Brown JE, Van Poznak C, Ibrahim T, Stemmer SM, Stopeck AT, Diel IJ, Takahashi S, Shore N, Henry DH, Barrios CH, Facon T, Senecal F, Fizazi K, Zhou L, Daniels A, Carriere P, Dansey R (2011) Incidence, risk factors, and outcomes of osteonecrosis of the jaw: integrated analysis from three blinded active-controlled phase III trials in cancer patients with bone metastases. Ann Oncol 23:1341-1347

8. Hoff AO, Toth BB, Altundag K, Johnson MM, Warneke CL, Hu M, Nooka A, Sayegh G, Guarneri V, Desrouleaux K, Cui J, Adamus A, Gagel RF, Hortobagyi GN (2008) Frequency and risk factors associated with osteonecrosis of the jaw in cancer patients treated with intravenous bisphosphonates. J Bone Miner Res 23:826-836

9. Solomon DH, Mercer E, Woo SB, Avorn J, Schneeweiss S, Treister N (2013) Defining the epidemiology of bisphosphonate-associated 
osteonecrosis of the jaw: prior work and current challenges. Osteoporos Int 24:237-244

10. Migliorati CA, Saunders D, Conlon MS, Ingstad HK, Vaagen P, Palazzolo MJ, Herlofson BB (2013) Assessing the association between bisphosphonate exposure and delayed mucosal healing after tooth extraction. J Am Dent Assoc 144:406-414

11. Gerstenfeld LC, Sacks DJ, Pelis M, Mason ZD, Graves DT, Barrero M, Ominsky MS, Kostenuik PJ, Morgan EF, Einhorn TA (2009) Comparison of effects of the bisphosphonate alendronate versus the RANKL inhibitor denosumab on murine fracture healing. J Bone Miner Res 24:196-208

12. Cao Y, Mori S, Mashiba T, Westmore MS, Ma L, Sato M, Akiyama T, Shi L, Komatsubara S, Miyamoto K, Norimatsu H (2002) Raloxifene, estrogen, and alendronate affect the processes of fracture repair differently in ovariectomized rats. J Bone Miner Res 17:2237-2246

13. Li J, Mori S, Kaji Y, Mashiba T, Kawanishi J, Norimatsu H (1999) Effect of bisphosphonate (incadronate) on fracture healing of long bones in rats. J Bone Miner Res 14:969-979

14. Lindsay R, Zhou H, Cosman F, Nieves J, Dempster DW, Hodsman AB (2007) Effects of a one-month treatment with PTH(1-34) on bone formation on cancellous, endocortical, and periosteal surfaces of the human ilium. J Bone Miner Res 22:495-502

15. Neer RM, Arnaud CD, Zanchetta JR, Prince R, Gaich GA, Reginster JY, Hodsman AB, Eriksen EF, Ish-Shalom S, Genant HK, Wang O, Mitlak BH (2001) Effect of parathyroid hormone (1-34) on fractures and bone mineral density in postmenopausal women with osteoporosis. N Engl J Med 344:1434-1441

16. Kwon YD, Lee DW, Choi BJ, Lee JW, Kim DY (2012) Short-term teriparatide therapy as an adjunctive modality for bisphosphonaterelated osteonecrosis of the jaws. Osteoporos Int 23:2721-2725

17. Bashutski JD, Eber RM, Kinney JS, Benavides E, Maitra S, Braun TM, Giannobile WV, McCauley LK (2010) Teriparatide and osseous regeneration in the oral cavity. N Engl J Med 363:2396-2405

18. Abtahi J, Agholme F, Sandberg O, Aspenberg P (2012) Bisphosphonateinduced osteonecrosis of the jaw in a rat model arises first after the bone has become exposed. No primary necrosis in unexposed bone. J Oral Pathol Med 41:494-499

19. Sonis ST, Watkins BA, Lyng GD, Lerman MA, Anderson KC (2009) Bony changes in the jaws of rats treated with zoledronic acid and dexamethasone before dental extractions mimic bisphosphonaterelated osteonecrosis in cancer patients. Oral Oncol 45:164-172

20. Reagan-Shaw S, Nihal M, Ahmad N (2008) Dose translation from animal to human studies revisited. FASEB J 22:659-661

21. Kuroshima S, Al-Salihi Z, Yamashita J (2013) Parathyroid hormone related to bone regeneration in grafted and nongrafted tooth extraction sockets in rats. Implant Dent 22:71-76

22. Kuroshima S, Go VA, Yamashita J (2012) Increased numbers of nonattached osteoclasts after long-term zoledronic acid therapy in mice. Endocrinology 153:17-28

23. Yamashita J, Koi K, Yang DY, McCauley LK (2011) Effect of zoledronate on oral wound healing in rats. Clin Cancer Res 17:1405-1414

24. Enlow DH (1966) Osteocyte necrosis in normal bone. J Dent Res 45:213

25. Bonnet N, Lesclous P, Saffar JL, Ferrari S (2013) Zoledronate effects on systemic and jaw osteopenias in ovariectomized periostin-deficient mice. PLoS One 8:e58726

26. McDonald MM, Dulai S, Godfrey C, Amanat N, Sztynda T, Little DG (2008) Bolus or weekly zoledronic acid administration does not delay endochondral fracture repair but weekly dosing enhances delays in hard callus remodeling. Bone 43:653-662

27. Peter CP, Cook WO, Nunamaker DM, Provost MT, Seedor JG, Rodan GA (1996) Effect of alendronate on fracture healing and bone remodeling in dogs. J Orthop Res 14:74-79

28. Allen MR, Chu TM, Ruggiero SL (2013) Absence of exposed bone following dental extraction in beagle dogs treated with 9 months of high-dose zoledronic acid combined with dexamethasone. J Oral Maxillofac Surg 71:1017-1026

29. Watts NB, Diab DL (2010) Long-term use of bisphosphonates in osteoporosis. J Clin Endocrinol Metab 95:1555-1565

30. McMillan MD (1975) An ultrastructural study of the relationship of oral bacteria to the epithelium of healing tooth extraction wounds. Arch Oral Biol 20:815-822

31. Ravanelli A, J, K (2006) Cranifofacial development. Lippincott Williams \& Wikins, Philadelphia

32. Eames BF, Helms JA (2004) Conserved molecular program regulating cranial and appendicular skeletogenesis. Dev Dyn 231:4-13

33. Aghaloo TL, Kang B, Sung EC, Shoff M, Ronconi M, Gotcher JE, Bezouglaia O, Dry SM, Tetradis S (2011) Periodontal disease and bisphosphonates induce osteonecrosis of the jaws in the rat. J Bone Miner Res 26:1871-1882

34. Aguirre JI, Akhter MP, Kimmel DB, Pingel JE, Williams A, Jorgensen M, Kesavalu L, Wronski TJ (2012) Oncologic doses of zoledronic acid induce osteonecrosis of the jaw-like lesions in rice rats (Oryzomys palustris) with periodontitis. J Bone Miner Res 27:2130-2143

35. Lopez-Jornet P, Camacho-Alonso F, Martinez-Canovas A, MolinaMinano F, Gomez-Garcia F, Vicente-Ortega V (2011) Perioperative antibiotic regimen in rats treated with pamidronate plus dexamethasone and subjected to dental extraction: a study of the changes in the jaws. J Oral Maxillofac Surg 69:2488-2493

36. Li D, Gromov K, Proulx ST, Xie C, Li J, Crane DP, Soballe K, O'Keefe RJ, Awad HA, Xing L, Schwarz EM (2010) Effects of antiresorptive agents on osteomyelitis: novel insights into the pathogenesis of osteonecrosis of the jaw. Ann N Y Acad Sci 1192:84-94

37. Aspenberg P, Genant HK, Johansson T, Nino AJ, See K, Krohn K, Garcia-Hernandez PA, Recknor CP, Einhorn TA, Dalsky GP, Mitlak BH, Fierlinger A, Lakshmanan MC (2010) Teriparatide for acceleration of fracture repair in humans: a prospective, randomized, doubleblind study of 102 postmenopausal women with distal radial fractures. J Bone Miner Res 25:404-414

38. Yamashita J, Datta NS, Chun YH, Yang DY, Carey AA, Kreider JM, Goldstein SA, McCauley LK (2008) Role of Bcl2 in osteoclastogenesis and PTH anabolic actions in bone. J Bone Miner Res 23:621-632

39. Nakajima A, Shimoji N, Shiomi K, Shimizu S, Moriya H, Einhorn TA, Yamazaki M (2002) Mechanisms for the enhancement of fracture healing in rats treated with intermittent low-dose human parathyroid hormone (1-34). J Bone Miner Res 17:2038-2047

40. Ettinger B, San Martin J, Crans G, Pavo I (2004) Differential effects of teriparatide on BMD after treatment with raloxifene or alendronate. J Bone Miner Res 19:745-751

41. Jilka RL, Weinstein RS, Bellido T, Roberson P, Parfitt AM, Manolagas SC (1999) Increased bone formation by prevention of osteoblast apoptosis with parathyroid hormone. J Clin Invest 104:439-446

42. Abtahi J, Agholme F, Aspenberg P (2013) Prevention of osteonecrosis of the jaw by mucoperiosteal coverage in a rat model. Int J Oral Maxillofac Surg 42:632-636 